\title{
Development of Portable Quartz Crystal Microbalance for Biosensor Applications
}

\author{
Jinxing Liang, ${ }^{*}$ Jing Zhang, ${ }_{1}^{1}$ Peng Wang, ${ }^{1}$ Changchuan Liu, ${ }^{1}$ Shi Qiu, ${ }^{1}$ and Toshitsugu Ueda ${ }^{2}$ \\ ${ }^{1}$ Key Laboratory of Micro-Inertial Instrument and Advanced Navigation Technology, \\ Ministry of Education, School of Instrument Science and Engineering, \\ Southeast University, 2 Sipailou, Xuanwuqu, Nanjing 210096, China \\ ${ }^{2}$ Graduate School of Information, Production and System, Waseda University, \\ Kitakyushu, Fukuoka 808-0135, Japan
}

(Received November 18, 2015; accepted February 29, 2016)

Keywords: quartz crystal microbalance, flow injection analysis, high frequency, biosensor

In this work, we report a novel flow-injection-type portable quartz crystal microbalance (QCM) sensor utilizing a high-frequency (higher than $40 \mathrm{MHz}$ ) miniaturized QCM chip. The resonator chip is assembled in a novel three-layer flow cell, in which the top and bottom layers are used to fix the spring-pin leading electrodes and the middle layer is used to house the resonator chip. A two-stage operational transconductance-amplifier-(OPA660)-based oscillator circuit is developed to drive the QCM and measure the frequency shift. A low human IgG concentration of $1 \mathrm{ppm}$ has been measured even using the simple physical adsorption antibody immobilization method, which indicates that the developed portable QCM sensor can be used as a biosensor in liquids.

\section{Introduction}

Quartz crystal microbalance (QCM) and surface plasmon resonance (SPR) sensors have been well known as transducers in the field of biosensors for a long time, owing to the merit of labelfree assay for immunological reagents. ${ }^{(1-4)}$ A specific membrane should be coated on the surfaces of the QCM and SPR sensors. The QCM converts the mass of adsorbed analyte into frequency shift, whereas the SPR measures the change in resonance angle induced by the biological reaction. Although the physical transduction principle is different between the two types of sensor, the biosensing mechanism is the same. These sensors have been extensively studied for applications in security and safety, such as explosive detection, ${ }^{(5-10)}$ food analysis, ${ }^{(11)}$ and environmental pollution monitoring. ${ }^{(12)}$ The two transducers can be combined with a flow injection analysis system, which makes the measurement more rapid and lessens the amount of analyte required. The two types of transducer have a similar resolution in the biosensing field and could also be made in multichannel mode, in which one channel is used as reference to compensate the liquid-property-induced signal fluctuation. In comparison with the mechanical structure, the QCM is much simpler than the SPR. The sensing element of the QCM is only one AT cut quartz chip coated with $\mathrm{Au} / \mathrm{Cr}$ metal films on two sides, and the SPR needs a light source and a light detection system aside from an optical prism. The simple structure of the QCM and the development of a quartz micro-electromechanical ${ }^{*}$ Corresponding author: e-mail: j-liang@seu.edu.cn 
system (MEMS) technique make it possible to achieve a miniaturized high-frequency QCM chip and resonator array. ${ }^{(13-16)}$

Recently, the fabrication and evaluation of a high-frequency fundamental QCM and the establishment of a portable flow-injection-based miniaturized QCM system have been reported in our previous publications. ${ }^{(17,18)}$ The QCM system is composed of a microsyringe pump, a sample injector, a tailor-made flow cell, and a frequency monitoring tool. Until now, measurements on a QCM chip in air or liquid are all performed with the aid of an impedance analyzer. The impedance analyzer could measure much information on the QCM resonator; however, it has a low frequency resolution owing to the wide scanning range. In this research, progress on the realization of a highfrequency oscillator, which is necessary for actual applications, and a more user-friendly flow cell will be reported. Finally, the working performance of the developed portable QCM is examined on the basis of the common immunoreaction between human IgG and anti-human IgG.

\section{Materials and Methods}

\subsection{Materials}

The high-frequency QCM chip is prepared by using our well-established wet etching process, and the fabrication and chip characterization have been reported in Refs. 17 and 18. The QCM chip dimensions are 5 (length) $\times 5$ (width) $\times 0.1$ (thickness) $\mathrm{mm}^{3}$. The fundamental frequency is higher than $40 \mathrm{MHz}$, and the quality factor is larger than 20000 in air and 1000 in pure water. The human IgG (whole molecule) and goat anti-human IgG (unconjugated) were purchased from Jackson Research (USA). The bovine serum albumin (BSA, protease-free) was purchased from MP Biomedicals (New Zealand).

\subsection{Flow cell}

Reference 18 provides the details of the established QCM measurement system, in which the flow cell is the key part to realize the flow injection analysis. The flow cell is composed of a cover and a substrate, and spring pins are used to lead the QCM electrodes into the impedance analyzer in a contact manner. The upper and lower spring pins are fixed into the cover and the substrate, respectively. The double-layer flow cell works well if the QCM chip is properly equipped. In fact, the fragile QCM chip was frequently destroyed when it was assembled into the flow cell. This is because the lower spring pin is located in the square groove, which is used to keep the QCM chip into the substrate, and the initial spring force by the spring pin makes the QCM chip unstable. Accordingly, when the cover is aligned to the substrate, the QCM chip is often destroyed. To solve this problem, a new three-layer flow cell is proposed. Figure 1 shows a schematic of the proposed flow cell, in which a middle layer is inserted between the cover and the substrate. The upper and lower spring pins are fixed into the cover and the substrate, respectively. The QCM chip is assembled by the following procedure. At first, the QCM is put into the square groove of the middle layer. Secondly, the cover is aligned and connected with the middle layer with two bolts. Finally, the substrate is aligned and connected with the middle layer with other two bolts. This assembly method provides a comfortable way of fixing the QCM chip while preventing the two spring pins from coming into contact with the chip at the same time. All of the three parts of the flow cell are made using polymethyl methacrylate (PMMA) glasses, which are useful for observing the assembly condition. 


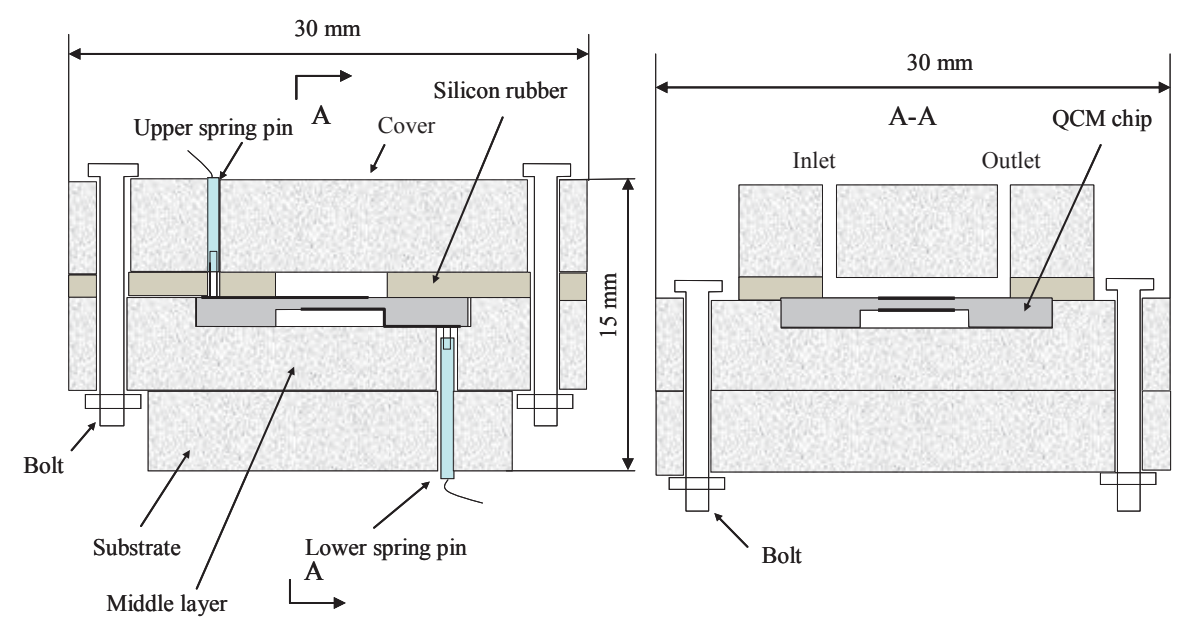

Fig. 1. (Color online) Schematic of the new three-layer flow cell.

\subsection{Oscillator circuit}

Although impedance or network analyzers could characterize the quartz resonator with more information such as the $Q$ value and equivalent circuit parameters besides resonance frequency, an oscillator circuit should be a common tool for actual sensor applications owing to its highfrequency measurement accuracy, portability, and easy operation. Generally, an oscillator circuit should satisfy the following two conditions to maintain stable oscillation, as shown in the following equations: ${ }^{(19)}$

$$
\begin{gathered}
|K A|=1, \\
\varphi=0,2 \pi, \ldots, 2 n \pi,
\end{gathered}
$$

where $K$ and $A$ represent the open loop gain and the feedback factor, respectively. Equations (1) and (2) indicate the amplitude and phase conditions for maintaining a stable oscillation, respectively.

When one face of the QCM chip is exposed to the liquid, the resonance quality factor sharply drops and the motional resistance markedly increases owing to the load of liquid viscosity.(18) Furthermore, the phase angle is also reduced to be very small even below $0^{\circ}$ with the increased fundamental frequency and density-viscosity values. ${ }^{(13)}$ To drive such a resonator, a two-stage transconductance-amplifier-(OPA660)-based oscillator circuit is designed, as shown in Fig. 2. The transconductance amplifier is composed of a voltage-controlled current source (VCCS) and a buffer amplifier. The first amplifier is used for oscillation and the quartz resonator is coupled with the emitter terminal. The second amplifier is used for power amplification and impedance matching $(50 \Omega)$. To meet the oscillation conditions shown in Eqs. (1) and (2), the zero-phase shift should be adopted by selecting the appropriate $\mathrm{C}_{17}$ and $\mathrm{RW}_{3}$, and the adjustable resistance $\mathrm{RW}_{1}$ is used to satisfy the amplitude condition, namely, $|K A|=1$. The diodes $\mathrm{D}_{1}$ and $\mathrm{D}_{2}$ provide input protection for the buffer amplifier. The network of the capacitance $\mathrm{C}_{8}$ and the inductance $\mathrm{L}_{1}$ works as a band-pass filter, and the center frequency is set at the QCM fundamental frequency. Through the coupling capacitance 


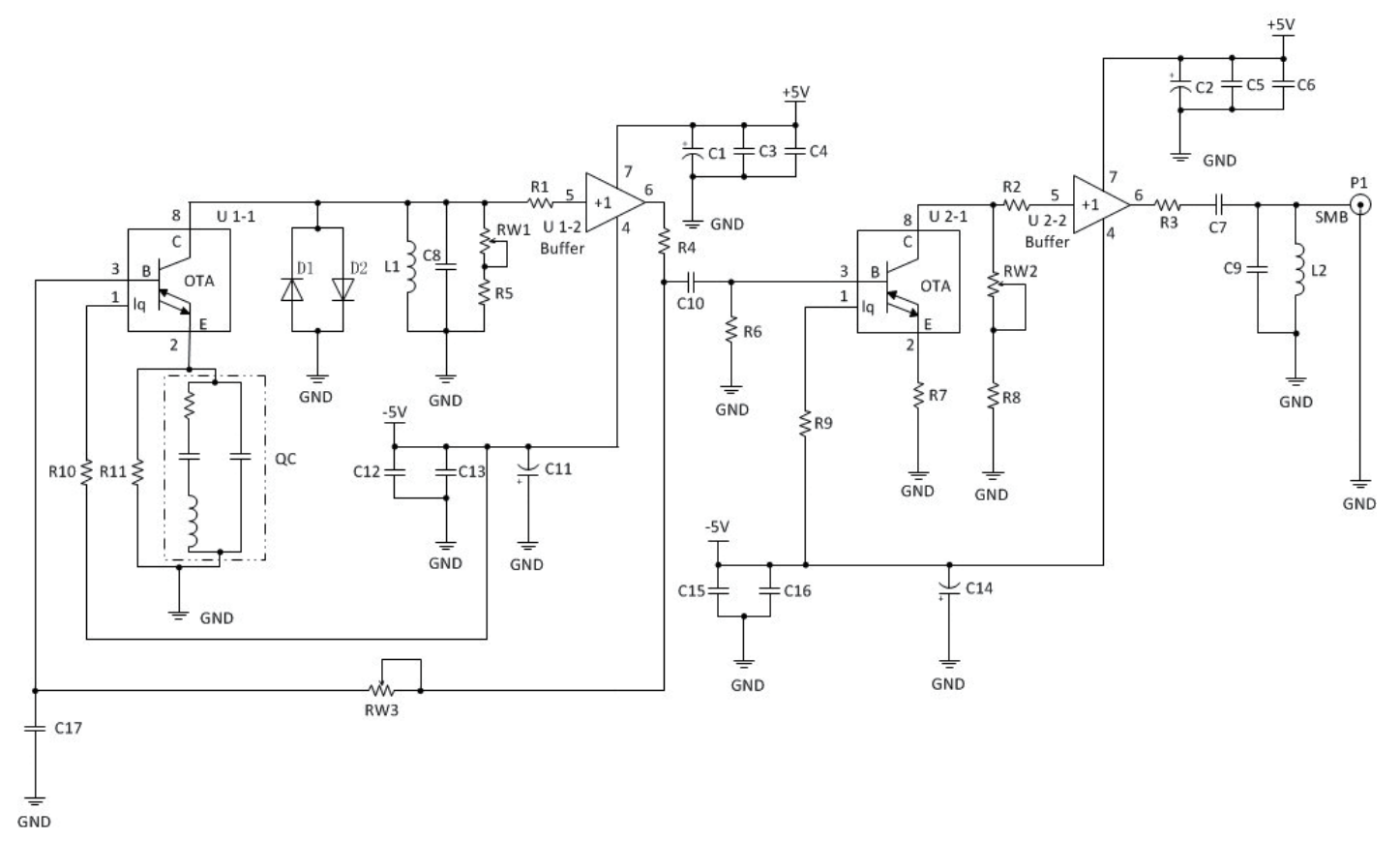

Fig. 2. High-frequency oscillator circuit for QCM in liquid.

$\mathrm{C}_{10}$ and $\mathrm{R}_{6}$, the signal is input into the second amplifier. At the output end, the capacitance $\mathrm{C}_{9}$ and the inductance $\mathrm{L}_{2}$ are also used as a band-pass filter, and the center frequency is set at the QCM resonance frequency. The frequency output is measured using the Agilent frequency counter 53132A and recorded with a PC.

\subsection{Examination as an immunosensor}

A simple antigen-antibody immunoassay is used to evaluate the established high-frequency QCM measurement system; the experimental flow is shown in Fig. 3. In detail, the QCM chip is cleaned in acetone solution for $15 \mathrm{~min}$, then rinsed in ethanol and pure water. After drying, the QCM chip is set into the flow cell, and a $0.1 \mathrm{mM}$ phosphate-buffered (PB, pH7.4) solution is allowed to flow through the QCM surface. When a stable frequency baseline is achieved, the antihuman IgG is injected to modify the sensor gold surface based on the simple physical adsorption. Then, the BSA solution is allowed to flow with the aim of blocking the unbound gold surface to avoid the possible nonspecific adsorption when measuring the analyte. Finally, human IgG solutions with different concentrations are injected to evaluate the detection limit. All the reagents are diluted using the PB solution.

\section{Results and Discussion}

After establishing the QCM measurement system, the frequency shift and stability affected by the flow rates are investigated. Figure 4 shows the measured results on a $49 \mathrm{MHz}$ QCM chip, and the flow rates (PB solution) were $1,5,10$, and $20 \mu 1 / \mathrm{min}$. It can be clearly seen that the oscillation frequency decreased with the increase in flow rate. Moreover, the frequency fluctuation is increased 


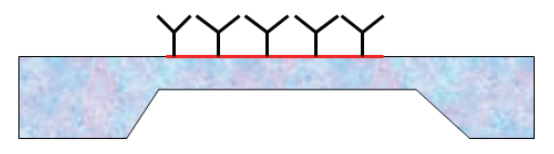

1) Immobilization of anti-human $\mathrm{IgG}$ on $\mathrm{Au}$ film

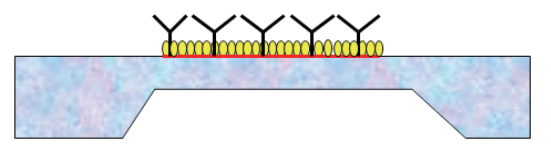

2) Blocking unbound surface with BSA

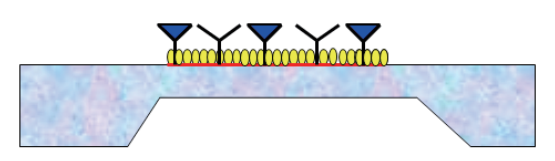

3) Binding of human IgG

$Y$ Anti-human IgG $\nabla$ Human IgG 0 BSA

Fig. 3. (Color online) Schematic of the experimental flow.

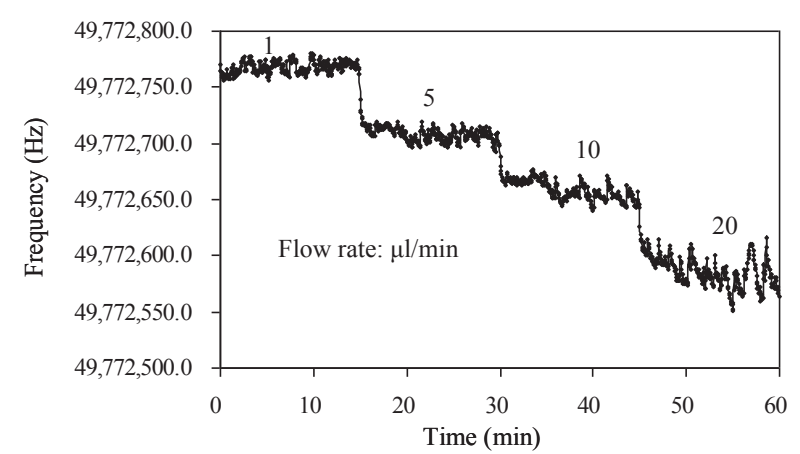

Fig. 4. Dependences of frequency and stability on flow rate.

with increasing flow rate, which is considered caused by the higher pump pressure and hydrostatic force at a higher flow rate. This result agrees well with a previous report. ${ }^{(16)}$ The standard deviation of frequency depending on the flow rates is summarized in Table 1. Furthermore, by considering a sufficient reaction antigen volume and reaction time, the flow rate of $10 \mu \mathrm{l} / \mathrm{min}$ is selected for the following immunoreaction experiment.

Figure 5 shows the antibody-antigen immunoreaction results investigated using our established portable high-frequency QCM system. For preparing the antibody-modified sensor surface, $20 \mathrm{ppm}$ anti-human IgG was injected twice and about $1000 \mathrm{~Hz}$ frequency shift was recorded. After that, 100 ppm BSA was injected twice to block the unbound gold surface, and the frequency drop of about $1600 \mathrm{~Hz}$ was observed, as shown in Fig. 5(a). Figures 5(b) and 5(c) show the immunoreaction experimental results. At first, the human IgG was injected in a continuous manner without surface regeneration, and the concentrations of the injected human $\operatorname{IgG}$ were 5, 20, 50, and $100 \mathrm{ppm}$. The accumulated frequency shifts were recorded in Fig. 5(b), which seems that the immobilized antibody membrane is more sensitive to low-concentration human IgG solution. Then, another experiment was performed to investigate the responses for several low concentrations using a separate fresh chip modified with the same antibody membrane. The concentrations of the injected human IgG were 1, 5, and $10 \mathrm{ppm}$. Even for the lowest concentration of $1 \mathrm{ppm}$, the frequency shift of about $150 \mathrm{~Hz}$ was clearly observed, as shown in Fig. 5(c). These results clearly demonstrated that the developed portable QCM system could properly work as a biosensor. This experiment mainly aimed to demonstrate that the developed QCM sensor could be used as a biosensor. In real applications, the lowest detection limit should be improved by using more effective antibody immobilization methods, such as modification via the well-known self-assembly monolayers (SAMs) and protein $\mathrm{G}$ or A to increase the antibody binding capability. Currently, we are focusing on improving the oscillator circuit to reduce the frequency fluctuation. 
Table 1

Standard deviation depending on flow rate.

\begin{tabular}{lcccc}
\hline Flow rate $(\mu \mathrm{l} / \mathrm{min})$ & 1 & 5 & 10 & 20 \\
\hline Standard deviation $(\mathrm{Hz})$ & 5.35 & 6.36 & 8.65 & 13.6 \\
\hline
\end{tabular}

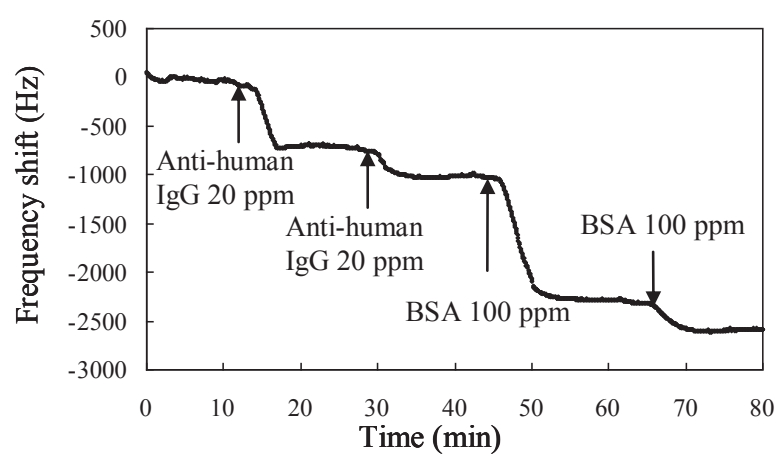

(a)

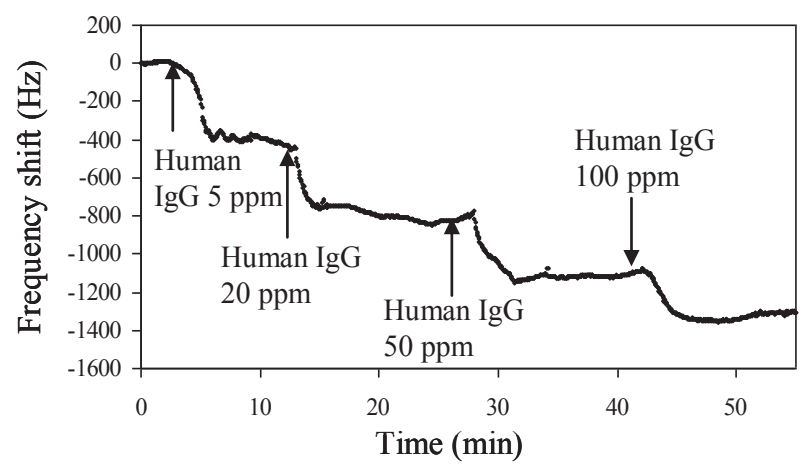

(b)

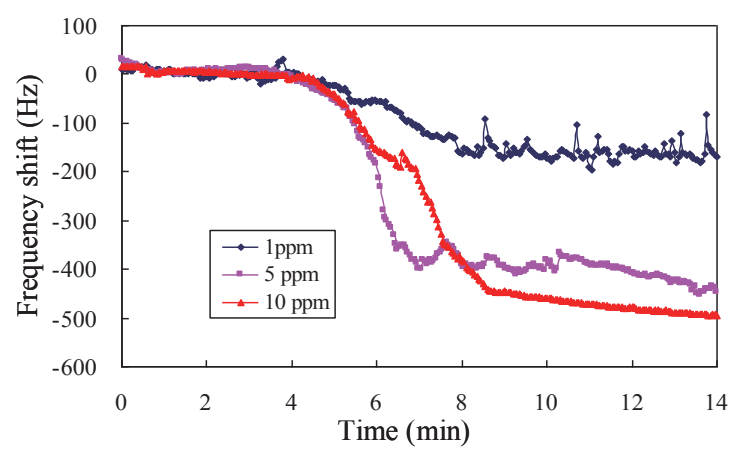

(c)

Fig. 5. (Color online) Measured frequency shift due to immunoreaction: (a) immobilization of anti-human IgG; (b) continuous injection of human IgG at concentrations of 5, 20,50, and $100 \mathrm{ppm}$; and (c) injection of human IgG at concentrations of 1,5 , and $10 \mathrm{ppm}$.

\section{Conclusions}

A portable miniaturized high-frequency QCM sensor has been developed. The QCM chip has a high fundamental frequency (higher than $40 \mathrm{MHz}$ ) with a small size below $5 \times 5 \mathrm{~mm}^{2}$. A tailormade three-layer flow cell and a high-frequency oscillator circuit have also been designed and fabricated. The application of the developed QCM sensor has been demonstrated by utilizing the human IgG and anti-human IgG reaction. Even by the simple physical adsorption antibody immobilization method, 1 ppm human IgG concentration could be clearly measured. 


\section{Acknowledgements}

This work was financially supported by the Scientific Research Foundation for the Returned Overseas Chinese Scholars, the State Education Ministry (the 46th), the Foundation of Key Laboratory of Micro-Inertial Instrument and Advanced Navigation Technology, Ministry of Education, China (201401), the Key Project of NSF of Jiangsu Province (BE2012740), and the Natural Science Foundation of Jiangsu Province (BK20130636).

\section{References}

1 C. KijBlinger, E. Uttenthaler, S. Drost, F. Aberl, H. Wolf, G. Brink, A. Stanglmaier, and E. Sackmann: Sens. Actuators, B 24-25 (1995) 107.

2 E. Yilmaz, D. Majidi, E. Ozgur, and A. Denizli: Sens. Actuators, B 209 (2015) 714.

3 D. A. White, A. K. Buell, C. M. Dobson, M. E. Welland, and T. P. J. Knowles: FEBS Lett. 583 (2009) 2587.

4 L. Sun, S. Svedhem, and B. Åkerman: Langmuir 30 (2014) 8432.

5 T. Onodera and K. Toko: Sensors 14 (2014) 16586.

6 R. Yatabe, T. Onodera, and K. Toko: Sens. Mater. 25 (2013) 45.

7 T. Onodera, T. Shimizu, N. Miura, K. Matsumoto, and K. Toko: IEEJ Trans. Sens. Micromach. 130 (2010) 269.

8 M. Procek, A. Stolarczyk, T. Pustelny, and E. Maciak: Sensors 15 (2015) 9563.

9 D. C. Apodaca, R. B. Pernites, F. R. Del Mundo, and R. C. Advincula: Langmuir 27 (2011) 6768.

10 D. D. Erbahar, I. Gürol, V. Ahsen, Z. Z. öztürk, Z. Zafer, E. Musluoğlu, and M. Harbeck: Sens. Lett. 9 (2011) 745 .

11 F. Ricci, G. Volpe, L. Micheli, and G. Palleschi: Anal. Chim. Acta 605 (2007) 111.

12 N. L. Torad, M. Naito, J. Tatami, A. Endo, S. Y. Leo, S. Ishihara, K. C. W. Wu, T. Wakihara, and Y. Yamauchi: Chem. Asian J. 9 (2014) 759.

13 B. Zimmermann, R. Lucklum, P. Hauptmann, J. Rabe, and S. Büttgenbach: Sens. Actuators, B 76 (2001) 47.

14 T. Abe and M. Higuchi: IEEE Sens. J. 11 (2011) 86.

15 P. Kao, S. Doerner, T. Schneider, D. Allara, P. Hauptmann, and S. Tadigadapa: J. Microelectromech. Syst. 18 (2009) 522.

16 M. Michalzik, R. Wilke, and S. Büttgenbach: Sens. Actuators, B 111-112 (2005) 410.

17 J. Liang, J. Huang, T. Zhang, J. Zhang, X. Li, and T. Ueda: Sensors 13 (2013) 12140.

18 J. Liang, J. Huang, S. Ding, and T. Ueda: Sens. Mater. 25 (2013) 519.

19 R. Lucklum and F. Eichelbaum: Springer Ser. Chem. Sens. Biosens. 5 (2007) 3. 